\section{Internal jugular vein thrombosis and ovarian hyperstimulation syndrome}

\author{
D M Raw \\ M C Collins
}

J R Soc Med 2007;100:339-340

Ovarian hyperstimulation syndrome can cause significant morbidity; early recognition may help to avoid complications of this iatrogenically induced condition.

\section{CASE REPORT}

A 30-year-old female with right-sided neck swelling was referred by her general practitioner as an acute admission to the Ear Nose Throat (ENT) department.

She presented with a five-day history of sudden onset neck pain radiating down her right arm and swelling in the anterior triangle. Examination showed low-grade pyrexia and a tender smooth cervical swelling but no lymphadenopathy.

Her recent medical history included polycystic ovary syndrome (PCOS) warranting assisted conception, commenced three months earlier. At the time of this current admission she was seven weeks pregnant with a twin pregnancy.

During this admission, the patient was referred to the radiology department for an ultrasound examination of the neck. This showed an extensive thrombus of the right internal jugular vein without extension into axillary vein (Figure 1A and 1B). Ultrasound of the patient's lower limbs revealed no evidence of deep vein thrombosis.

The patient had a two-year history of infertility secondary to PCOS and had only recently commenced ovarian stimulation and intracytoplasmic sperm injection (ICSI) treatment for this, with subsequent embryo transfer. Four weeks prior to this admission she had been admitted under the care of gynaecologists with abdominal pain. Full blood count had shown a raised haemoglobin $(16.1 \mathrm{~g} / \mathrm{dL})$ and haematocrit (0.47), as well as thrombocytosis $\left(459 \times 10^{9} / \mathrm{L}\right)$; her coagulation screen was normal. Abdominal ultrasound showed moderate volume ascites, bilateral pleural effusions and an enlarged, multi-cystic right ovary $(15 \times 8 \mathrm{~cm})$ (Figure $2 \mathrm{~A}$ and $2 \mathrm{~B})$.

Department of Clinical Radiology, Royal Hallamshire Hospital, Sheffield Teaching Hospitals NHS Foundation Trust, Glossop Road, Sheffield S1O 2JF, UK

Correspondence to: Daniel M Raw, 60 Kensington Drive, Lodge Moor, Sheffield S1O 4NF, UK

Email: Rawdm4@hotmail.com (a)

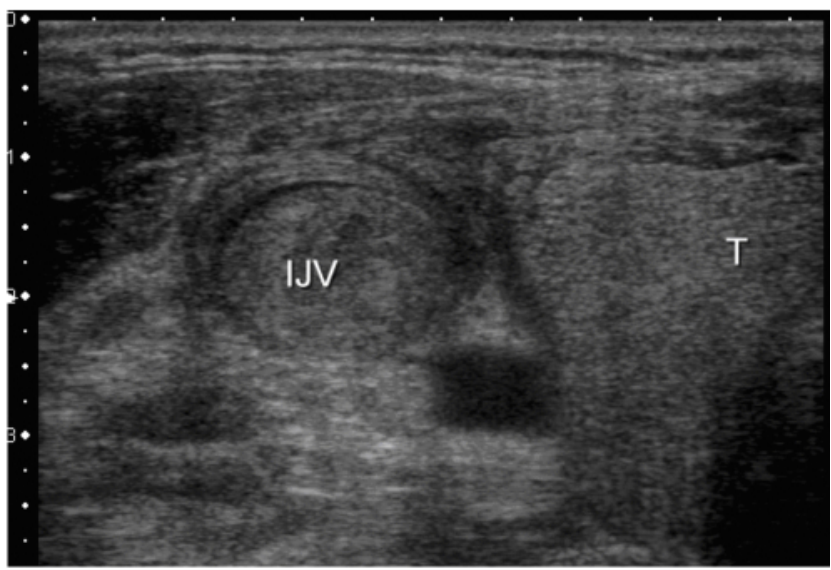

(b)

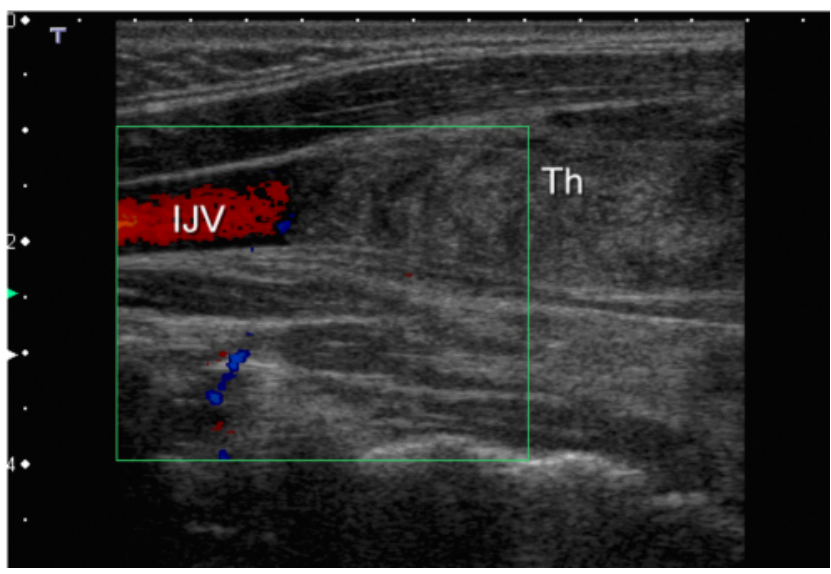

Figure 1 A: Ultrasound image in transverse plane at the level of the thyroid (T). The right internal jugular vein (IJV) is enlarged containing echogenic thrombus. B: Longitudinal ultrasound image showing flow above the level of thrombus (Th) on colour Doppler. Internal jugular vein (IJV). [In colour online.]

Her results were consistent with ovarian hyperstimulation syndrome, and she was started on conservative treatment, including two weeks of anti-coagulation therapy with low molecular weight heparin. This had been stopped a fortnight before the admission with the neck swelling.

In summary, this was a 30-year-old patient, seven weeks pregnant with a twin pregnancy following assisted conception. She was diagnosed with a right internal jugular vein thrombosis secondary to ovarian hyperstimulation syndrome. Treatment was with low molecular weight heparin, to be reviewed post partum.

\section{DISCUSSION}

Ovarian hyperstimulation occurs in all women who have received gonadotrophins to induce ovulation in assisted conception regimes. It usually manifests as a mild form. This needs to be distinguished from the clinical entity of 
(a)

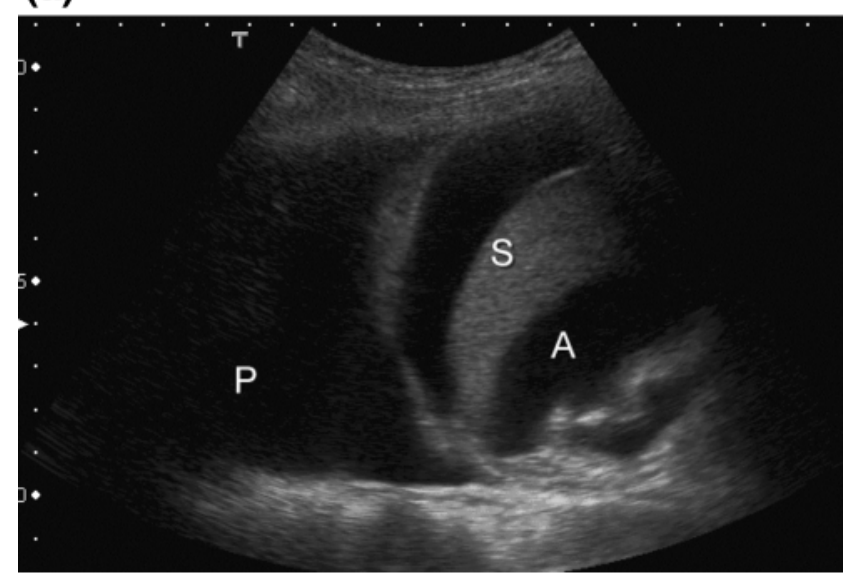

(b)

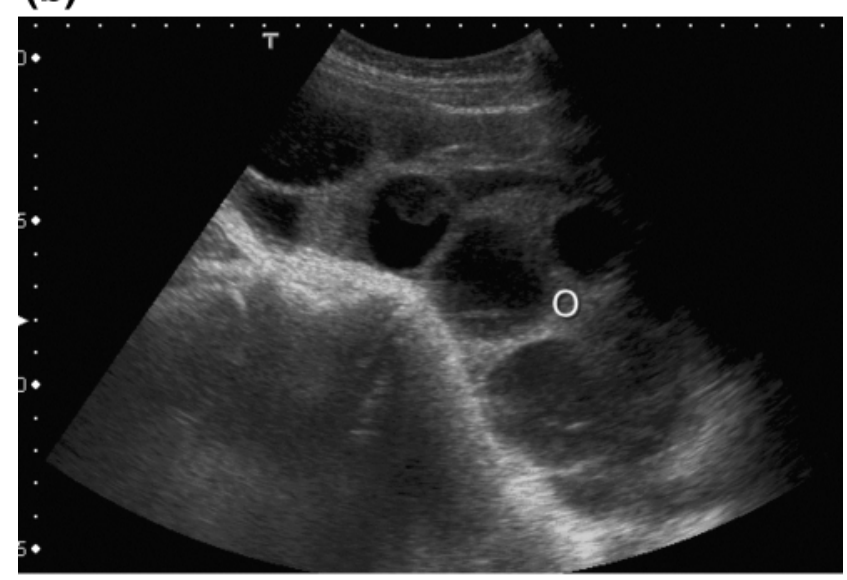

Figure 2 A: Longitudinal ultrasound image of the left upper quadrant of the abdomen showing moderate pleural effusion (P) and ascites (A) surrounding the spleen (S). B: Longitudinal ultrasound image of the pelvis showing a markedly enlarged right ovary (0).

ovarian hyperstimulation syndrome (OHSS), which represents a major complication of ovulation induction. ${ }^{1,2}$

OHSS in its severest form causes marked ovarian enlargement due to the formation of multiple cysts, ascites and pleural effusions. ${ }^{1,4}$ The hypovolaemia and haemoconcentration which follow can lead to serious complications, including hypovolaemic shock, acute renal failure, thromboembolic phenomenon and death. ${ }^{1,3,4}$ Common symptoms of OHSS are abdominal pain, distension and nausea.

There have been several classifications of OHSS since it was first recognized. The syndrome can generally be separated into mild, moderate and severe forms depending on clinical, biochemical and radiological features. The mild form is thought to be of no clinical relevance and is seen in most patients following ovarian stimulation. ${ }^{1}$ Incidence of moderate-to-severe OHSS is thought to occur in $1-10 \%$ of women undergoing ovulation induction. ${ }^{5}$
The pathophysiology of OHSS is poorly understood. It is thought to be due to vasoactive substances released by the ovaries in response to gonadotrophin stimulation, resulting in vascular leakage of fluid into the third space., ${ }^{2,6}$ Recognized risk factors are youth, low maternal weight, PCOS, high doses of exogenous gonadotrophins and previous episodes of OHSS..$^{1,4}$

The radiological features of OHSS include pleural effusions and bibasal atelectasis on chest radiography, and ascites, pleural effusions and massive enlargement of ovaries with multiple cysts on ultrasonography. ${ }^{1,7}$

Complications are thought to be secondary to hypovolaemia and the hypercoaguable state induced by increased vascular permeability. High levels of oestrogen and relative venous stasis in the lower limb secondary to ovarian enlargement are also contributory. ${ }^{3,5}$ Thromboembolic events can affect the venous or arterial systems and are potentially lethal. ${ }^{1,5}$ In comparison to the general population, thromboembolic phenomenon are more commonly seen in the upper body - the upper limbs, the neck and intracranially. ${ }^{3,5}$ Treatment of choice for thromboembolic events is anticoagulation but the OHSS needs treating itself. 3,5

Infertility treatment is becoming commonplace in today's society. In light of this, complications are likely to be more frequently encountered - and not just within the practice of infertility physicians. Awareness of imaging findings and the clinical condition will aid early recognition of OHSS and should expedite diagnosis and management of what is fundamentally an iatrogenic condition.

\section{Competing interests None declared.}

\section{REFERENCES}

1 Aboulghar M, Mansour R. Ovarian hyperstimulation syndrome: classifications and critical analysis of preventative measures. Hum Reprod Update 2003;9:275-89

2 Elchalal U, Schenker J. The pathophysiology of ovarian hyperstimulation syndrome - views and ideas. Hum Reprod 1997;12:1129-37

3 Stewart J, Hamilton P, Murdoch A. Upper limb thrombosis associated with assisted conception treatment. Hum Reprod 1997;12:2174-5

4 Practice committee of the American society for reproductive medicine. Ovarian Hyperstimulation Syndrome. Fertil Steril 2003;80:1309-14

5 Ou Y, Kao YL, Lai SL, et al. Thromboembolism after ovarian stimulation: successful management of a women with superior sagittal thrombosis after IVF and embryo transfer: case report. Hum Reprod 2003; 18:2375-81

6 Delbaere A, Smits G, De Leener A, Costagliola S, Vassart G. Understanding ovarian hyperstimulation syndrome. Endocrine 2005;26: 285-90

7 McNeary M, Stark P. Radiographic findings in Ovarian Hyperstimulation Syndrome. J Thorac Imaging 2002;17:230-2 